\title{
Recycling von minderwertigen Aluminiumschrotten
}

\author{
Stefan Wibner und Helmut Antrekowitsch \\ Lehrstuhl für Nichteisenmetallurgie, Montanuniversität Leoben, Leoben, Österreich \\ Eingegangen 19. Januar 2021; angenommen 8. Februar 2021; online publiziert 4. März 2021
}

\begin{abstract}
Zusammenfassung: Aluminiumschrott ist häufig mit einer Vielzahl an Stoffen verunreinigt, wie Kunstoffen, Dichtungen, Ölen und Holz, oder liegt in lackierter bzw. beschichteter Form vor. Dabei handelt es sich um organische Bestandteile, welche in den Schmelzöfen zu Reaktionen und damit zum Verlust von metallischem Aluminium führen. Im Rahmen dieses Beitrags erfolgt eine Erläuterung der industriell üblichen Verfahren zur Verarbeitung von minderwertigen Al-Schrotten, welche einen hohen Organikanteil aufweisen. Typischerweise kommen diese in Refinern zum Einsatz, wo Schmelzsalze Anwendung finden. Dabei erfolgt die Herstellung von Gusslegierungen, wozu auch Desoxidationsgranalien, welche in der Stahlherstellung Verwendung finden, zählen. Eine weitere Möglichkeit zur Erzeugung dieser Produkte bietet der Schmelzprozess im Zweikammerofen, wo durch Pyrolyse vorab ein hoher Anteil organischer Bestandteile entfernt werden kann. Im experimentelIen Teil des Beitrags finden Laboruntersuchungen statt, um Schmelzausbeuten von gemischtem Al-Schrott mit hohem Organikgehalt zu bestimmen. Hierbei werden die metallurgischen Rahmenbedingungen dahingehend eingestellt, dass eine Vergleichbarkeit mit den industriell eingesetzten Schmelzaggregaten gegeben ist. Die besten Ergebnisse konnten dabei durch die Anwendung von Schmelzsalzen erreicht werden. Bei der Verarbeitung minderwertiger Schrotte im Zweikammerofen führt eine vorgeschaltete Pyrolyse zur Erhöhung der Schmelzausbeuten und zur Verringerung der Krätzemengen.
\end{abstract}

Schlüsselwörter: Recycling, Aluminium, Organische Anteile, Schmelzausbeuten

\footnotetext{
$\overline{\text { Dipl.-Ing. S. Wibner ( } \triangleleft)}$

Lehrstuhl für Nichteisenmetallurgie,

Montanuniversität Leoben,

Franz-Josef-Straße 18,

8700 Leoben, Österreich

stefan.wibner@unileoben.ac.at
}

Recycling of Low-grade Aluminium Scrap

Abstract: Aluminium scrap is often contaminated with a wide variety of materials, such as plastics, seals, oils, and woods, or it exists in a varnished form. These are organic compounds, which cause reactions in aluminium melting furnaces and lead to the loss of metallic Al. In the theoretical part of this work, an explanation of the customary industrial processes for recycling of Al scrap with a high organic content is given. Typically, it is processed in refiners where molten salts are used for the production of casting alloys, including deoxidation granules that are used in steel production. The melting process in a twochamber furnace with pyrolysis in the scrap chamber offers a further possibility for producing these granules, whereby organic constituents can be removed partially before the actual melting of the scrap. In the experimental part, laboratory trials are carried out to determine the metal yields of mixed Al scrap with a high organic content. The basic metallurgical conditions are set in such a way that they can be compared with the industrial melting units. The best results were achieved by using molten salts for the recycling process. For the processing of contaminated scraps in a two-chamber furnace, a previous pyrolysis also leads to an increase in metal yields and to a reduction in the amount of formed dross.

Keywords: Recycling, Aluminium, Organic content, Metal yield

\section{Allgemeines}

Beim Aluminiumrecycling sind zwei grundlegende Verfahrensvarianten zu unterscheiden. Umschmelzwerke (Remelter) dienen der Produktion von Knetlegierungen durch den Einsatz wenig verunreinigter Schrotte. Stärker kontaminierte Materialien, zu denen minderwertige Al-Schrotte zählen, welche oftmals einen hohen Organikanteil aufweisen, gelangen unter Verdünnung mit Reinaluminium und $\mathrm{Zu}$ satz von Salzen in Schmelzhütten (Refiner), wo Gusslegierungen hergestellt werden. Nachfolgend wird zuerst auf 
Abb. 1: Verfahrensablauf beim Recycling von Aluminium mit Salzschlacke. (laut Beschreibungen nach Krone [1])

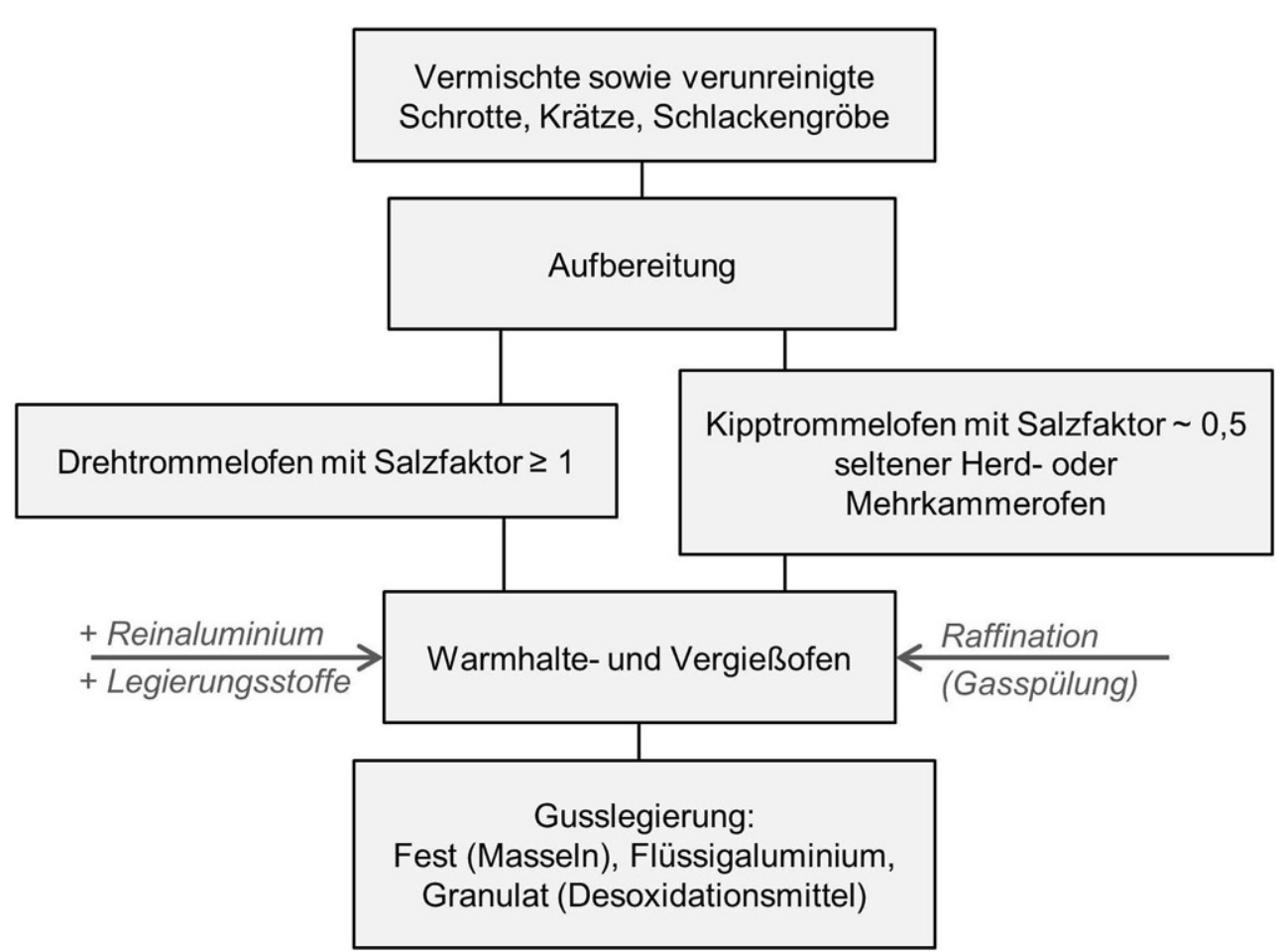

die Verfahrensroute in Refinern eingegangen, besonders im Hinblick auf die dort verwendeten Schmelzaggregate. Da für die Verarbeitung minderwertiger Schrotte vor allem die Pyrometallurgie unter der Anwendung von Schmelzsalz von Bedeutung ist, werden auch einige wesentliche Grundlagen zum Salzbadschmelzen erläutert [1, 2].

\subsection{Recyclingroute zur Verarbeitung minderwer- tiger Aluminiumschrotte}

Das typische Prozessschema der Refiner für das Recycling vermischter und verunreinigter Al-Schrotte, welche oftmals einen hohen Organikgehalt aufweisen, sowie hoch-oxidhaltiger Materialien ist in Abb. 1 dargestellt [1].

Als übliche Technologie in diesem Zusammenhang ist das Salzbadschmelzen im Drehtrommelofen hervorzuheben, da hierbei aufgrund der Anwendung von Schmelzsalz praktisch alle Schrotte bzw. aluminiumhaltigen Vorstoffe verarbeitet werden können. Als Unterscheidungskriterium zwischen Dreh- und Kipptrommelöfen ist der Salzfaktor zu nennen, welcher als das Verhältnis der Massen von Schmelzsalz zu nichtmetallischen Komponenten der Einsatzstoffe definiert ist und vom verwendeten Ofenaggregat abhängt. Dieser liegt beim Drehtrommelofen im Bereich zwischen 1,0-1,8. [1-4].

Das Schmelzen im Kipptrommelofen erfolgt vor allem für die Verarbeitung von oxidhaltigen Materialien, wie Krätzen oder Gröben aus der Salzschlackenaufarbeitung. Hierbei wird mit geringeren Mengen an Salz gearbeitet (Salzfaktor etwa 0,5), wodurch ein trockener, krümeliger Salzkuchen entsteht, der durch Kippen aus dem Ofen entfernt wird. Nach der Schmelzeraffination im Warmhalteofen erfolgt die
Herstellung von Gusslegierungen in Form von Masseln, Flüssigaluminium oder Granulat, wobei letzteres als Desoxidationsmittel in der Eisen- und Stahlerzeugung dient. Die anfallenden Salzschlackenmengen liegen im Bereich von $0,2-0,4$ t pro Tonne Recyclingaluminium, welche nachfolgend aufzuarbeiten sind. Hierbei werden das Salz und das darin enthaltene metallische Al rückgewonnen und Metalloxide bzw. andere inerte Oxidrückstände ausgetragen, wobei diese in der Zementindustrie Einsatz finden können [1-3].

Darüber hinaus kann auch der Zweikammerofen als Alternative zu den Salzschmelzverfahren verwendet werden, um Desoxidationsaluminium aus minderwertigen Al-Einsatzstoffen zu erzeugen. Die Anwendung dieser Technologie findet beispielsweise bei der Hütte Klein-Reichenbach in Niederösterreich statt. Bei dem dort vorhandenen Schmelzaggregat handelt es sich um einen Closed-Well-Herdofen, der im Wesentlichen aus einem Vorherd als Schrottschmelzkammer und einer Heizkammer besteht (siehe Abb. 2). Diese zwei Herde sind über Hubtüren, welche der gesamten Breite des Ofens entsprechen, zugänglich. Die Umwälzung der Schmelze erfolgt mittels elektromagnetischer Pumpe, wobei in der Prinzipskizze laut Abb. 2 auch ein kleiner offener Chargierherd zum Einschmelzen feinteiliger Schrotte existiert $[1,4,5]$.

Das Chargieren von unterschiedlich stark verunreinigten Schrotten erfolgt in die Schrottkammer, wo das Vorwärmen und Abschwelen stattfinden, sodass sich Lacke und Kunststoffe verflüchtigen. Mit den nachfolgenden Schrottchargen werden die vorhergehenden, bereits vorgewärmten und abgeschwelten Einsatzstoffe in das Schmelzbad geschoben und schmelzen von unten ab. Das dabei entstehende Gas wird über eine Schwelgasleitung in die Heiz- 
Abb. 2: Schematische Darstellung eines Zweikammerofens [6]

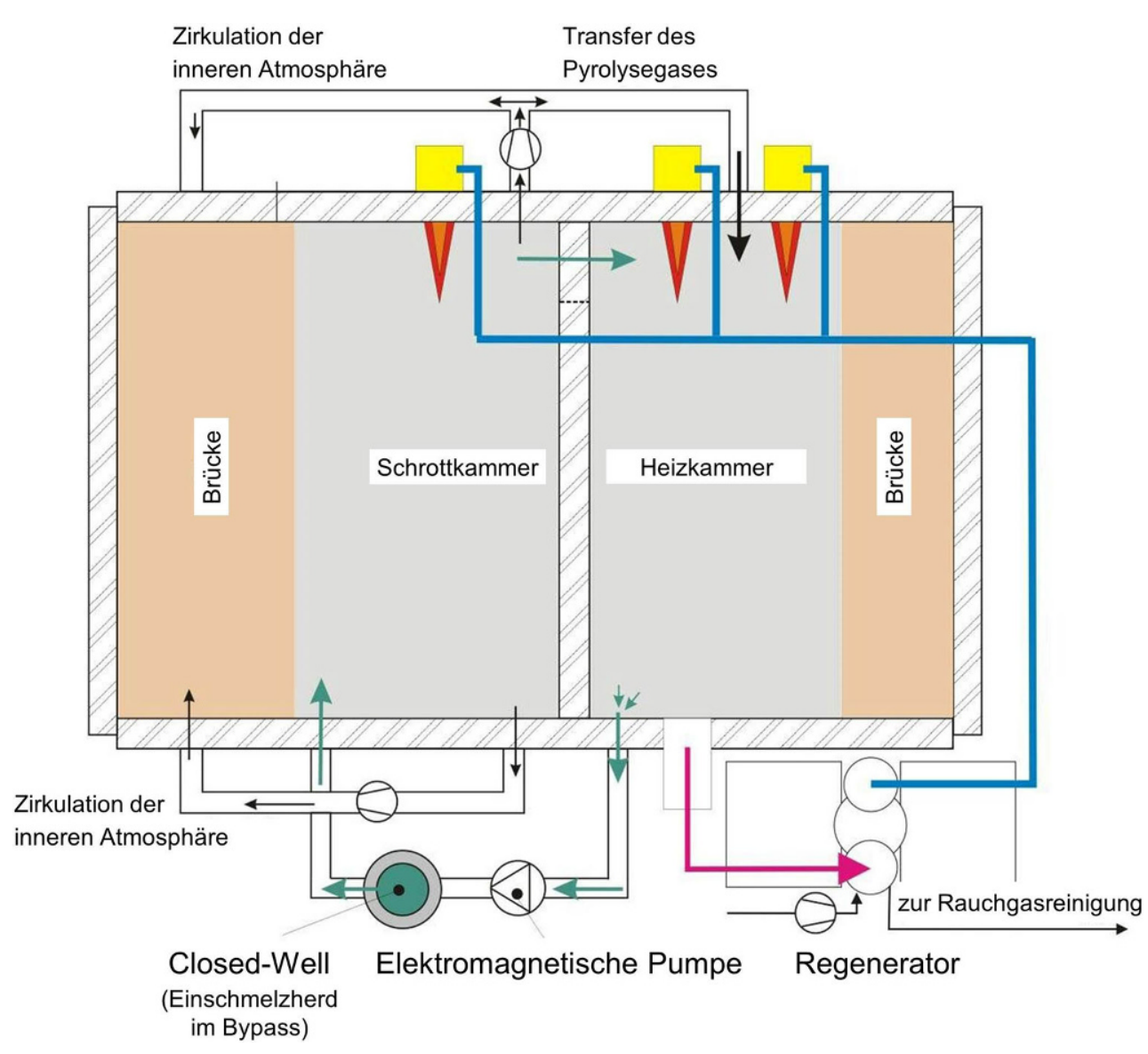

Die Aufgaben des Schmelzsalzes lassen sich allgemein in vier Punkten zusammenfassen und sind in der Standardliteratur wie folgt formuliert: $[1,4]$

- Aufbrechen der Oxidhäute

- Aufnahme der nichtmetallischen Verunreinigungen und aufgebrochenen Oxidhäute

- Förderung der Koagulation von Metalltropfen zur Bildung einer Schmelze

- Abdecken der Schmelze zum Schutz vor weiterer Oxidation

Die Zusammensetzung der Salzmischung variiert je nach Einsatzgebiet, Schrottart und Ofentyp. Insgesamt existieren mehr als 100 unterschiedliche Mischungen, welche in der Al-Recyclingindustrie Anwendung finden. In Europa wird hinsichtlich der enthaltenen Chloride meist ein Gemisch aus $70 \% \mathrm{NaCl}$ und $30 \% \mathrm{KCl}$ verwendet. In den USA und Kanada hingegen findet die äquimolare eutektische Mischung aus $50 \mathrm{~mol}-\% \mathrm{NaCl}$ und $50 \mathrm{~mol}-\% \mathrm{KCl}$ Einsatz $[1,4,7,8]$.

Der Mechanismus des Ablösens der Oxidschicht beruht auf der Tatsache, dass die Grenzflächenspannung zwischen Salzschmelze und Oxid geringer ist als jene zwischen Oxid und Aluminium. Durch den Zusatz von Fluoriden kommt es zur lokalen Anlagerung dieser oberflächenaktiven Elemente, was zur Herabsetzung der Grenzflächenspannung zwischen Salz und Aluminium führt. Der daraus entstehende Spannungsgradient erhöht die Kraft zur Abtrennung. Nach 

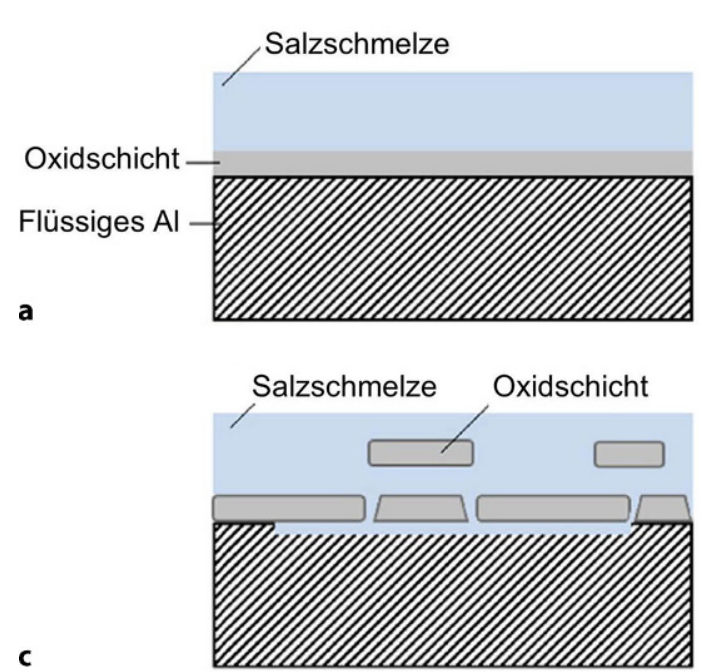

b
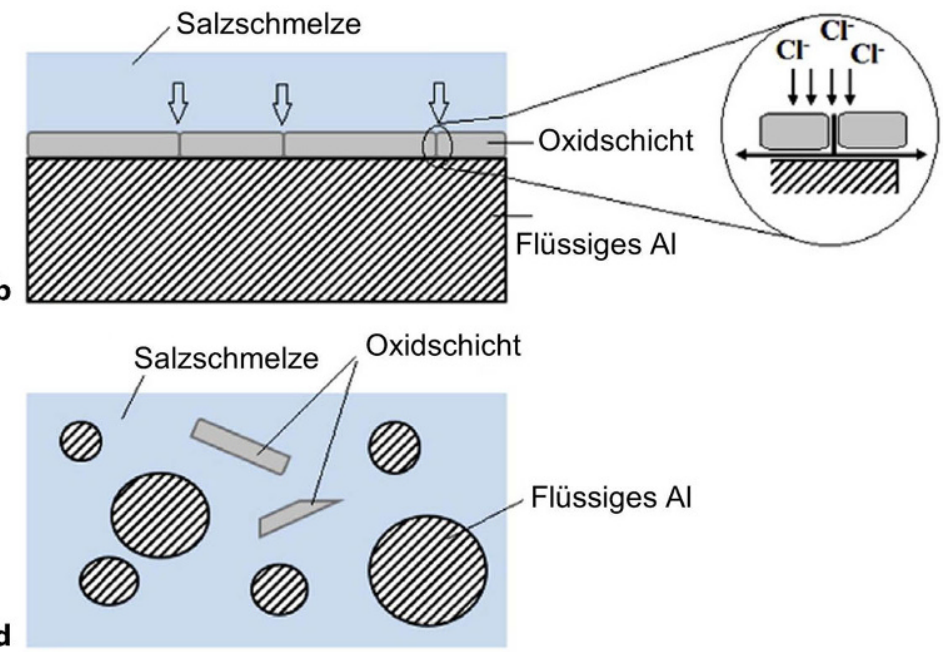

Abb. 3: Schematische Darstellung der Oxidschichtabtrennung beim Recycling von Aluminium unter Anwendung von Schmelzsalz [8]

Mahinroostaa und Allahverdi [8] spielen auch die Chloranionen im Schmelzsalz eine wesentliche Rolle beim Angriff der Oxidschicht. Die Darstellung der Phasen der Oxidfilmablösung erfolgt in Abb. 3, wobei

a. den Kontakt von Salzschmelze und Oxidschicht,

b. den Angriff des Oxidfilms durch Chloride,

c. die Oxidabtrennung durch Eindringen des flüssigen Salzes und

d. die beiden separierten Phasen, flüssiges Aluminium und Oxidpartikel,

darstellen $[4,7,8]$.

Grundlage für die Phasenseparation zwischen der sich bildenden Salzschlacke und der Aluminiumschmelze ist eine ausreichend hohe Dichtedifferenz. Des Weiteren stellt die Viskosität der Schlacke einen entscheidenden Parameter dar, welcher die Koagulation der Al-Tröpfchen beeinflusst. Beide Einflussgrößen hängen vor allem von der Zusammensetzung, dem Oxidgehalt und der Temperatur der Salzmischung ab $[1,4,9]$.

\section{Experimentelle Untersuchungen}

Im experimentellen Teil dieses Beitrags werden Schmelzversuche von gemischtem, lackiertem Al-Schrott, der geschreddert vorliegt und einen hohen Organikgehalt aufweist, durchgeführt, um aufzuzeigen, dass bei entsprechender Fahrweise des Ofenaggregates auch minderwertige Vorstoffe ohne Salz zur Erzeugung von Granalien verwertet werden können. Dabei finden drei Experimente mit den maximalen Massen an Einsatzstoffen statt, welche am Lehrstuhl für Nichteisenmetallurgie im Labormaßstab verarbeitet werden können. Dies ist erforderlich, um möglichst repräsentative Schrotteinsatzmengen gewährleisten zu können. Ziel ist es, sowohl den Verfahrensablauf im Refiner als auch jenen im Zweikammerofen, welche beide zuvor erläutert wurden, abzubilden und die Schmelzausbeuten des Schrottes bzw. die sich bildenden Krätzemengen zu ermitteln. Daher erfolgt beim ersten Versuch das Umschmelzen des Schrottes unter Anwendung von Schmelzsalz. Bei den weiteren beiden Experimenten wird eine flüssige AlSchmelze im Tongrafittiegel vorgelegt, in welche der ver-

Abb. 4: Krätzebildung am AlSchmelzbad: V2-lackiert (a), V3-pyrolysiert (b)
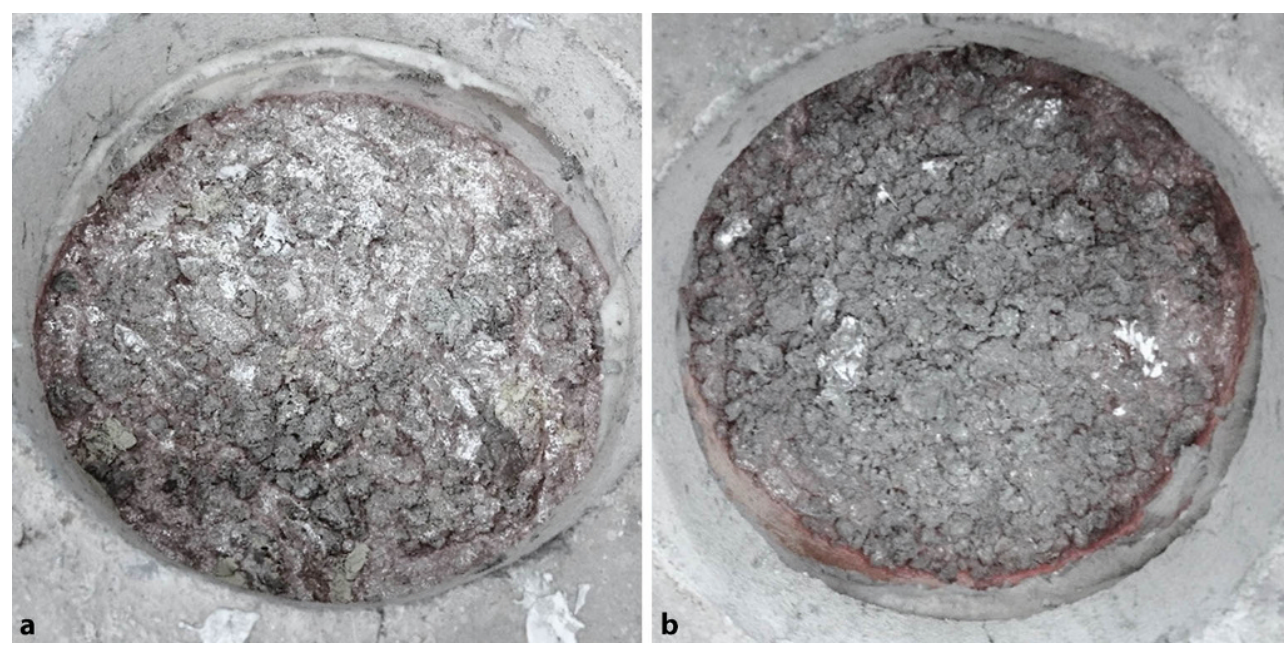


\begin{tabular}{|c|c|c|c|}
\hline \multicolumn{4}{|c|}{\begin{tabular}{|l} 
TABELLE 1 \\
Massenbilanz zu den Schmelzversuchen
\end{tabular}} \\
\hline & V1 & V2 & V3 \\
\hline \multicolumn{4}{|l|}{\begin{tabular}{|l} 
Einwaage $[\mathrm{kg}]$ \\
\end{tabular}} \\
\hline Al-Vorlage & - & 79,81 & 79,57 \\
\hline Schrott lackiert & 50,00 & 20,00 & 20,00 \\
\hline Schmelzsalz & 37,50 & - & - \\
\hline \multicolumn{4}{|l|}{ Pyrolyseverlust } \\
\hline Gasförmig [\%] & - & - & 1,02 \\
\hline Fest [\%] & - & - & 0,34 \\
\hline Schrott pyrolysiert [kg] & - & - & 19,73 \\
\hline \multicolumn{4}{|l|}{ Rückwaage $[\mathrm{kg}]$} \\
\hline Krätze & - & 2,48 & 2,03 \\
\hline Al metallisch & 46,08 & 97,27 & 97,32 \\
\hline \multicolumn{4}{|c|}{ Ergebnisse (bezogen auf Schrott lackiert) } \\
\hline Schmelzausbeute [\%] & 92,16 & 87,30 & 88,75 \\
\hline Krätze [\%] & - & 12,40 & 10,15 \\
\hline
\end{tabular}

unreinigte Schrott chargiert wird, wobei dieser bei einem Versuch lackiert und beim zweiten pyrolysiert vorliegt.

\subsection{Schmelzexperiment unter Anwendung von Schmelzsalz}

Für die experimentelle Untersuchung des Schmelzprozesses entsprechend einem Refiner erfolgt ein Umschmelzen der repräsentativen Schrottprobe unter Schmelzsalz, wobei hier die europäische Salzmischung bestehend aus 70\% $\mathrm{NaCl}, 30 \% \mathrm{KCl}+2 \% \mathrm{CaF}_{2}$ Anwendung findet (V1). Als Parameter werden eine Temperatur von ca. $800^{\circ} \mathrm{C}$ und eine Schmelzzeit von etwa 30 min gewählt, da dies auch den typischen Bedingungen bei Probeschmelzen im Rahmen der Schrottbemusterung entspricht (laut Krone [1]). Das Verhältnis der Schrott-zu Salzmassen beträgt 1:0,75. Aufgrund der begrenzten Größe des Ofenaggregates und damit des Tongrafittiegels werden $50 \mathrm{~kg}$ Schrott und $37,5 \mathrm{~kg}$ Schmelzsalz verwendet. Die Zugabe des Schrottes erfolgt erst, nachdem die Salzmischung flüssig vorliegt. Nach Beendigung des Schmelzvorgangs fallen als Produkte metallisches Aluminium in Barrenform sowie Salzschlacke an, wobei letztere noch einen geringen Anteil an Aluminium in Form fein verteilter Partikel enthält. Um diese rückzugewinnen, erfolgt das Mahlen und Sieben der Schlacke. Anschließend findet eine Aufteilung in Korngrößenbereiche statt, wobei es sich bei $>1 \mathrm{~mm}$ sowie bei $0,5-1 \mathrm{~mm}$ um metallisches $\mathrm{Al}$ handelt. Dieses wird mit warmem Wasser gelaugt und getrocknet, um anhaftende Salzschlackenreste zu entfernen. Das Feingut stellt die spröd-brechende Schlacke ohne metallischen Al dar.

\subsection{Schmelzexperimente unter Vorlage einer flüssigen Al-Schmelze}

Insgesamt erfolgt die Durchführung von zwei Versuchen, bei denen als Einsatzstoff wiederum gemischter, geschred- derter Al-Schrott dient, welcher beim ersten Experiment in lackierter Form (V2) und beim zweiten im pyrolysierten Zustand (V3) vorliegt. Die Pyrolyse findet dabei in einem geschlossenen Ofenaggregat bei $600^{\circ} \mathrm{C}$ für $12 \mathrm{~h}$ unter Stickstoffatmosphäre statt. Beim Schmelzprozess im Kipptiegelofen wird Primäraluminium, mit einer Masse von $80 \mathrm{~kg}$, als Vorlage in Form von Masseln in den Tongrafittiegel chargiert und in den schmelzflüssigen Zustand überführt. Nachfolgend erfolgt die Zugabe von etwa $20 \mathrm{~kg}$ Schrott. Die genauen Massen der unterschiedlichen Einsatzstoffe sind in Tab. 1 angegeben. Die Schrottchargierung findet in fünfminütigen Abständen statt, wobei hier jeweils $1 \mathrm{~kg}$ unter zusätzlichem manuellem Tauchen mittels Tauchglocke zugegeben wird. Die Temperatur des Schmelzbades ist ständig zu kontrollieren und sollte zwischen 720 und $750^{\circ} \mathrm{C}$ liegen. Diese Versuchsbedingungen ermöglichen die optimale Abbildung des industriellen Prozesses im Zweikammerofen. Eine vollständige Pyrolyse in der Schrottkammer wäre hierbei anzustreben, welche allerdings aufgrund unterschiedlicher Einflussparameter (Schrottart, Feuchtigkeit, Temperatur, Ofenmannschaft etc.) nicht immer möglich ist. Das Schmelzexperiment mit dem pyrolysierten Schrott als Einsatzstoff bildet einen optimalen Abschwelprozess in der Schrottkammer ab, jenes mit dem lackierten Material zeigt das Worst-Case-Szenario. Abschließend erfolgen ein Abziehen der gebildeten Krätze (siehe Abb. 4) und das Abgießen des flüssigen Aluminiums. Erstere entsteht aufgrund der hohen Affinität von Al zu Sauerstoff und enthält neben $\mathrm{Al}_{2} \mathrm{O}_{3}$ auch Silizium-, Eisen-, Kalzium- und Magnesiumoxide sowie andere Aluminiumverbindungen (Karbide, Chloride, Fluoride) in geringen Mengen. Des Weiteren ist zu erwähnen, dass in der Krätze noch ein hoher Anteil an metallischem Al, oftmals bis zu $60 \%$, enthalten ist, dieser allerdings beim Schmelzprozess im Zweikammerofen nicht direkt genutzt werden kann und daher auch bei den Experimenten im Labormaßstab dahingehend keine näheren Untersuchungen stattfinden.

\subsection{Auswertung der Schmelzexperimente}

In Tab. 1 sind die Ergebnisse der drei Schmelzversuche zusammengefasst. Bei V1 ergibt sich ein metallischer Al-Inhalt von $92,16 \%$ bezogen auf die Schrotteinwaage, wobei die in der Salzschlacke enthaltenen Al-Partikel bereits mitberücksichtigt sind. Dieses Ergebnis entspricht somit dem maximalen rückgewinnbaren Al-Inhalt.

Bei V2 ergeben sich eine Schmelzausbeute von $87,30 \%$ und eine Krätzemenge von $12,40 \%$. Durch eine vorhergehende Pyrolyse, wie dies bei V3 erfolgt ist, lässt sich erstere auf $88,75 \%$ erhöhen und es bildet sich weniger Krätze. Bei Betrachtung des industriellen Prozesses im Zweikammerofen erfolgt eine Abtrennung der gasförmigen Pyrolyseprodukte, eine Abscheidung der festen findet allerdings nicht statt. Daher wird der - nach dem Pyrolysevorgang abgesiebte und verwogene - Pyrolysekoks beim Schmelzprozess im Labormaßstab mit dem Schrott in das Schmelzbad chargiert. Die geringere Krätzemenge sowie die etwas höhere Schmelzausbeute sind auf den verringerten Organikgehalt zurückzuführen, wodurch Metallverluste 
durch Abbrand minimiert werden. Daraus lässt sich feststellen, dass ein möglichst vollständiger Abschwelvorgang in der Schrottkammer des industriell eingesetzten Zweikammerofens von wesentlicher Bedeutung zur Erzielung hoher Schmelzausbeuten und geringer Krätzemengen ist [10]. Aufgrund des in organischen Stoffen enthaltenen Kohlenstoffs kommt es in der Al-Schmelze zusätzlich zur exothermen Al-Karbidbildung, wodurch das produzierte Metall verunreinigt und die Oxidation von Aluminium gefördert wird [11].

\section{Zusammenfassung}

Im Rahmen des Beitrags erfolgte die Erläuterung von industriell eingesetzten Verfahren zum Recycling minderwertiger Al-Schrotte mit hohem Organikgehalt besonders im Hinblick auf die hierbei verwendeten Schmelzaggregate. Der experimentelle Teil beinhaltet die Durchführung von drei Schmelzversuchen, um sowohl den Prozess im Salzbadofen, als auch jenen im Zweikammerofen abzubilden und die Schmelzausbeuten von minderwertigem Al-Schrott vergleichend zu bestimmen. Hier konnten die besten Ergebnisse bei der Verarbeitung unter Schmelzsalz erzielt werden, wobei die Aufarbeitung des Salzes als zusätzlicher Schritt zu sehen ist. Eine vorgeschaltete Pyrolyse führt darüber hinaus zur Erhöhung der Ausbeuten und zur Verringerung der Krätzemengen im Zweikammerofen.

Funding. Open access funding provided by Montanuniversität Leoben.

Open Access Dieser Artikel wird unter der Creative Commons Namensnennung 4.0 International Lizenz veröffentlicht, welche die Nutzung, Vervielfältigung, Bearbeitung, Verbreitung und Wiedergabe in jeglichem Medium und Format erlaubt, sofern Sie den/die ursprünglichen Autor(en) und die Quelle ordnungsgemäß nennen, einen Link zur Creative Commons Lizenz beifügen und angeben, ob Änderungen vorgenommen wurden.

Die in diesem Artikel enthaltenen Bilder und sonstiges Drittmaterial unterliegen ebenfalls der genannten Creative Commons Lizenz, sofern sich aus der Abbildungslegende nichts anderes ergibt. Sofern das betreffende
Material nicht unter der genannten Creative Commons Lizenz steht und die betreffende Handlung nicht nach gesetzlichen Vorschriften erlaubt ist, ist für die oben aufgeführten Weiterverwendungen des Materials die Einwilligung des jeweiligen Rechteinhabers einzuholen.

Weitere Details zur Lizenz entnehmen Sie bitte der Lizenzinformation auf http://creativecommons.org/licenses/by/4.0/deed.de.

\section{Literatur}

1. Krone, K.: Aluminiumrecycling: Vom Vorstoff bis zur fertigen Legierung, Düsseldorf: Vereinigung Deutscher Schmelzhütten e.V., 2000

2. Martens, H.; Goldmann, D.: Recyclingtechnik: Fachbuch für Lehre und Praxis, 2. Aufl., Wiesbaden: Springer Vieweg, 2016

3. Rombach, G.: Recycling aus der Sicht eines integrierten Aluminiumkonzerns - Rohstoffe, Technologie, Qualität, in: Thomé-Kozmiensky, K. J.; Goldmann, D. (Hrsg.): Recycling und Rohstoffe, Band 3, Neuruppin: TK-Verlag, 2010, S. 527-537

4. Schmitz, C.: Handbook of Aluminium Recycling, Essen: Vulkan-Verlag, 2006

5. Boin, U.: Stand der Technik in der Sekundäraluminiumerzeugung im Hinblick auf die IPPC-Richtlinie, Wien: Umweltbundesamt $\mathrm{GmbH}_{\text {, }}$ 2000. (Monographien, Band: 120)

6. LOI Thermoprocess GmbH: Funktionsprinzip des ZweikammerSchmelzofens, Essen: Technische Beschreibung TCF, 2014

7. Gisbertz K.; Friedrich, B.: VeMRec-Metallurgische Herausforderungen beim Recycling von NE-Metallkonzentraten aus Abfallverbrennungs-Rostasche, in: Thomé-Kozmiensky, K. J. (Hrsg.): Mineralische Nebenprodukte und Abfälle 2 - Aschen, Schlacken, Stäube und Baurestmassen, Neuruppin: TK-Verlag, 2015, S. 227-253

8. Mahinroosta, M.; Allahverdi, A.: Hazardous aluminum dross characterization and recycling strategies: A critical review, Journal of environmental management, 223 (2018), pp 452-468

9. Roy, R. R.; Ye, J.; Sahai, Y.: Viscosity and Density of Molten Salts Based on Equimolar $\mathrm{NaCl}-\mathrm{KCl}$, Materials Transactions, JIM, 38 (1997), pp 566-570

10. Wibner, S.; Antrekowitsch, H.: Recycling of aluminium with variation of the scrap processing techniques, Proceedings of European Metallurgical Conference (2019), Düsseldorf, Deutschland, 2019, pp 1151-1161

11. Antrekowitsch, H.; Paulitsch, H.; Pirker, A.: Einsatz minderwertiger Schrotte und Reststoffsituation beim Recycling von Aluminium, in Thomé-Kozmiensky, K. J.; Goldmann, D. (Hrsg.): Recycling und Rohstoffe, Band 6, Neuruppin: TK Verlag, 2013, S. 433-453

Hinweis des Verlags. Der Verlag bleibt in Hinblick auf geografische Zuordnungen und Gebietsbezeichnungen in veröffentlichten Karten und Institutsadressen neutral. 